
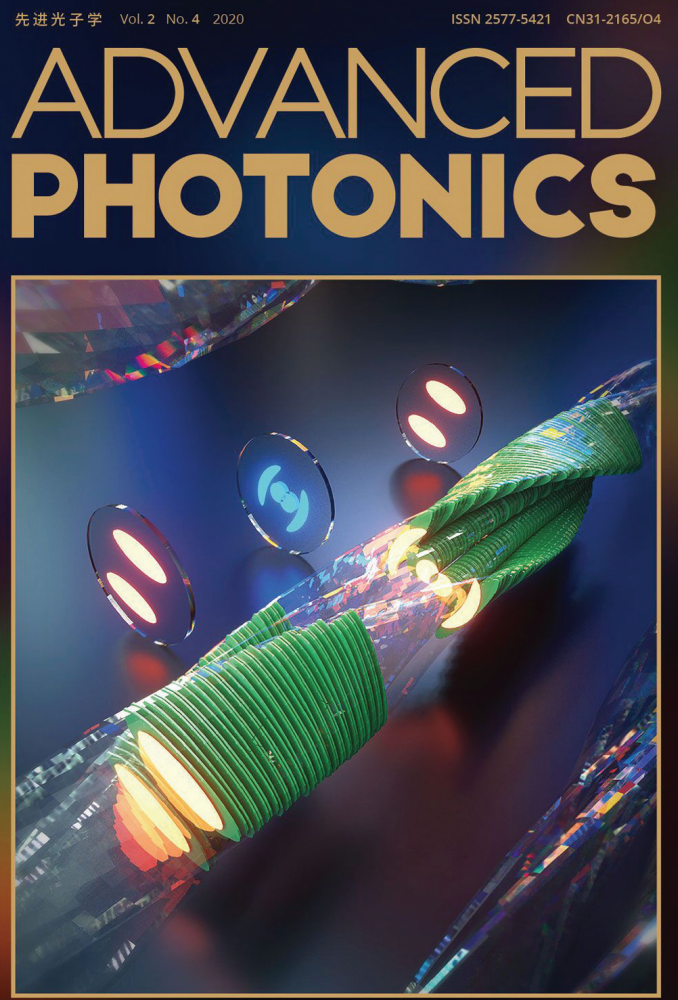

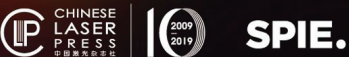

\section{About the cover: Advanced Photonics Volume 2, Issue 4}

The image on the cover for Advanced Photonics Volume 2, Issue 4, illustrates the Rabi oscillation of azimuthons in a weakly nonlinear circular waveguide with weakly longitudinally periodic modulated refractive index change. The image is based on the original research presented in the article by
Kaichao Jin, Yongdong Li, Feng Li, Milivoj Belic, Yanpeng Zhang, and Yiqi Zhang: "Rabi oscillations of azimuthons in weakly nonlinear waveguides," Adv. Photon. 2(4), 046002, doi: 10.1117/1.AP.2.4.046002. 